\title{
Effect of ultrasound on the aqueous viscosity of several water-soluble polymers
}

\author{
Josue Addiel Venegas-Sanchez, Motohiro Tagaya and Takaomi Kobayashi
}

The effect of ultrasound (US) on the shear viscosity of water-soluble polymers, polyvinyl alcohol, polyethylene glycol and polyacrylic acid, was studied in aqueous solutions with US exposure of $23,43,96$ and $141 \mathrm{kHz}$ for an $8.5-9 \mathrm{~W}$ US. The US exposure significantly decreased the shear viscosity of the solutions in this order: $43>23>96>141 \mathrm{kHz}$. When US exposure was stopped, the shear viscosities of the aqueous polymer solutions reverted to their original values. US stimulation during the decrease in viscosity was supported by the ultrasonic power transmitted through the aqueous polymer solution. In addition, Fourier-transform infrared spectra obtained during US exposure showed that hydrogen bonds in the aqueous polymer solution could be broken, especially at $43 \mathrm{kHz}$. We concluded that US exposure influenced hydrogen-bond interactions between the $\mathrm{OH}$ groups of the polymer and water molecules in the aqueous medium.

This finding was supported by US absorption of the aqueous polymer solution in the transmittance model, which shows the US absorptivity, $\varepsilon_{U S}$, for each polymer system.

Polymer Journal (2013) 45, 1224-1233; doi:10.1038/pj.2013.47; published online 15 May 2013

Keywords: hydrogen bonding; shear viscosity; ultrasound

\section{INTRODUCTION}

Ultrasound (US) has been widely applied in different fields, such as medicine, engineering and biology. ${ }^{1}$ Because of the advantages of US, it is widely used in sono-chemical processes ${ }^{2,3}$ as well as in food engineering and separation processes. ${ }^{4,5}$ Smart materials responded when external stimuli, such as heat, light or magnetic fields, were applied. ${ }^{6}$ However, few studies of US have demonstrated the ability of external stimuli to change material properties. Recently, it was reported that US could be used as a new type of trigger in the drug delivery of polymeric composite materials. In these cases, a low US frequency was applied to encapsulate drugs, and the hydrophilicity and high permeability of the materials improved the penetration of water. ${ }^{7,8}$ In our previous works, copolymer microgels of $\mathrm{N}$-isopropylacrylamide and acrylic acid using methylenebisacrylamide as a cross-linker demonstrated US-responsive behavior in spherical polymer microgels. ${ }^{9,10}$ In addition, a significant change in the shear viscosity was observed in $\mathrm{Al}_{2} \mathrm{O}_{3}$ /polyacrylic acid (PAA) slurry, ${ }^{11}$ and similar behavior was observed in $\mathrm{N}$-isopropylacrylamide copolymers with PAA in aqueous media ${ }^{12}$ and carrageenans. ${ }^{13}$ These variations were explained by the breaking of polymeric hydrogen bonds in the microenvironment when US exposure was performed in aqueous medium.

Regarding the changes in shear viscosity during US exposure, US absorption might induce polymer changes, particularly those related to hydrogen-bonded species in the aqueous polymers used. It was reported that ultrasonic adsorption to polymeric medium was evaluated by absorption coefficients, sound velocities and viscosity, for different nonionic and ionic polymer solutions, such as polyethylene glycol (PEG), polyvinyl alcohol (PVA) and PAA, at high frequencies of $2-130 \mathrm{MHz} .{ }^{14-18}$ However, no results for US using lower frequencies have been reported.

Nevertheless, water-soluble polymers possessing different chemical structures for PEG, PVA and PAA are widely used in different industries. These polymers are generally used as stabilizers for aqueous colloidal suspensions, and their properties in aqueous media are strongly related to the viscosity of their aqueous media. ${ }^{19-24}$ Therefore, if the viscosity could be controlled by US, such a technology would possess quite an effective advantage. However, the effect of US in polymer systems has not been well characterized when lower US frequencies are used. The present study, therefore, investigated the shear viscosity properties of the aqueous polymers of PVA, PEG and PAA as a function of ultrasonication at various low frequencies: 23, 43, 96 and $141 \mathrm{kHz}$. In addition, the effects of US in aqueous solutions were studied using a US absorption model and were also observed using Fourier-transform infrared (FT-IR) spectroscopy in aqueous solutions. It was concluded that absorption of US altered hydrogen bonding in the aqueous solution.

\section{EXPERIMENTAL PROCEDURE}

Materials and preparation of sample solution

Water-soluble polymers, PEG, PVA and PAA, were purchased from Sigma Aldrich Co., St Louis, MO, USA. The molecular weight of PEG was 


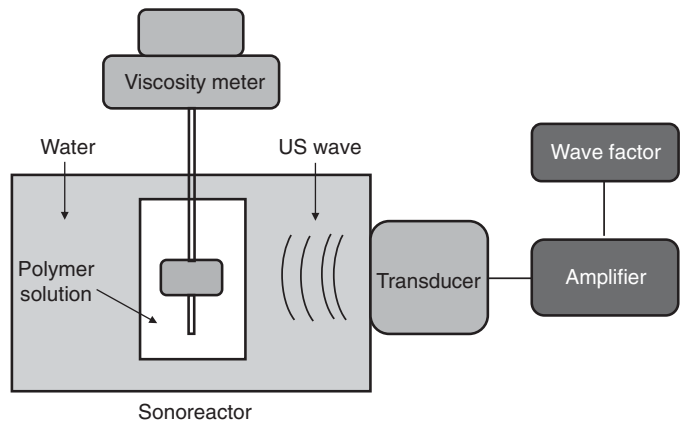

Scheme 1 US experimental setup for measuring the shear viscosity in polymer solutions.

$100000 \mathrm{Mw}$; the molecular weight of PVA was $146000-186000 \mathrm{Mw}$, with a degree of hydrolysis of approximately $86-88 \%$; and the molecular weight of PAA was $450000 \mathrm{Mw}$. An aqueous solution with a $10 \mathrm{wt} \%$ concentration for each polymer was prepared in distilled water and then purified using an ion exchange column. The polymer solutions were heated for $2 \mathrm{~h}$ to allow for full dissolution at $60^{\circ} \mathrm{C}$. The transparent solutions obtained were slowly cooled at room temperature and stored at that temperature for 1 day to allow for settling and to eliminate air bubbles.

\section{Shear viscosity measurements of the water-soluble polymers}

A sonoreactor device (HSR-305; Honda Electronics Co. Ltd., Toyohashi, Aichi, Japan) was used for the experiments (Scheme 1). The sonoreactor could be operated at four frequencies: 23, 43, 96 and $141 \mathrm{kHz}$. A calorimetric study was also performed to calculate the US power dissipated in the water bath. ${ }^{25,26}$ The power dissipated into the aqueous solution was between 8.5 and $9 \mathrm{~W}$. The measurement setup, as shown in Scheme 1, includes a Brookfield rotating viscometer (Tokyo Keiki Inc., Ohta-Ku, Tokyo, Japan) to measure the shear viscosity of the aqueous solutions. The sonoreactor temperature was fixed at $25^{\circ} \mathrm{C}$ for all experiments by flowing water through the sonoreactor. It was confirmed that no increase in temperature was detected during the experiments. The shear viscosity was measured both before and after US exposure. The viscosity was measured with a No. 2 rotor. The viscous solution was put into a cylindrical glass vessel with a diameter of $40 \mathrm{~mm}$ and height of $120 \mathrm{~mm}$ and was placed in the US sonoreactor. The sample solution was exposed to US for $5 \mathrm{~min}$ at each frequency. The shear viscosity was measured at 1-min intervals.

\section{Measurements of the US effect on the FT-IR spectra of the aqueous solutions}

FT-IR spectroscopy is a useful technique to examine the hydrogen bonding of polymeric materials. Accordingly, the US effect on chemical changes in watersoluble polymer solutions was analyzed by FT-IR spectroscopy, which was measured using an FT-IR 4100 series spectrometer (Jasco Corp., Hachioji Tokyo, Japan). Aqueous polymer solutions with a concentration of $10 \mathrm{wt} \%$ were lightly dropped onto a $\mathrm{CaF}_{2}$ window $(30 \mathrm{~mm}$ diameter, $2 \mathrm{~mm}$ thickness; Pier Optics Co. Ltd., Tatebayashi, Gunma, Japan). Another window was placed on top to cover the drop of solution, which formed a thin layer between the plates. To prevent penetration of water into the sample window, when being placed into the US water bath, the $\mathrm{CaF}_{2}$ windows were sealed with parafilm. The windows had no effect on polymer adsorption and no influence on the resulting FT-IR spectra. All experiments were performed three times and were shown to have similar results within an experiment. The FT-IR spectra were measured immediately using absorbance mode following 1,3 and $5 \mathrm{~min}$ of US exposure. For the FT-IR spectra, spectral analysis of the $\mathrm{OH}$ and $\mathrm{CH}$ stretching bands was deconvoluted using a method similar to that in our previous report, and these stretching bands ranged from 2400 to $3800 \mathrm{~cm}^{-1}$ for PAA and from 3000 to $3800 \mathrm{~cm}^{-1}$ for both PEG and PVA. ${ }^{13}$ When the centers of the fixed peaks of the original $\mathrm{OH}$ and $\mathrm{CH}$ stretching bands were determined in the spectral data, their bands were decomposed into Gaussian components with peak centers, and the curve was then fitted using OriginPro 8.5.1 (LightStone Corporation, Tokyo, Japan).

\section{RESULTS AND DISCUSSION}

Effect of USs on the shear viscosity of water-soluble polymers

The shear viscosity of the aqueous polymer solutions was measured after US exposure at $8.5-9 \mathrm{~W}$ for $5 \mathrm{~min}$, after which time ultrasonication was stopped and the shear viscosity was measured at 1-min intervals. Figure 1 shows the change in shear viscosity as a function of time for each aqueous solution of (a) PEG, (b) PVA and (c) PAA at $\mathrm{pH} 3$, (d) PAA at $\mathrm{pH} 5$ and (e) PAA at $\mathrm{pH}$ 10. Here PAA solutions were prepared at three $\mathrm{pH}$ values, 3,5 and 10, because the extent of dissociation of the $-\mathrm{COOH}$ group of PAA depends on the $\mathrm{pH}$. The concentration of each polymer was fixed at $10 \mathrm{wt} \%$ for the viscosity measurements. As observed previously, the shear viscosity apparently decreased from the original values following US exposure in each case. When the viscosity returned to its original value, each sample was exposed to US a second time for $5 \mathrm{~min}$. It was noted that the change in the shear viscosity during the second cycle appeared similar to that during the first cycle. Therefore, these results indicated that the US exposure could respond to the change in the shear viscosity. The results also indicated that the change in the shear viscosity was caused by the US stimulus, which was used as a trigger to induce a change in shear viscosity. Overall, the $43-\mathrm{kHz}$ frequency was the most effective stimulus for the shear viscosity comparison with the other frequencies. The decrease in the shear viscosity occurred in increasing order of $43>23>96>141 \mathrm{kHz}$. It would be interesting to consider that the comparison of the chemical structures of each polymer is made based on the viscosity change due to US. For the $43-\mathrm{kHz}$ sample, the PEG solution before US exposure was $543 \mathrm{cp}$ and after US exposure changed to $474 \mathrm{cp}$. For the PVA solution, a change in viscosity was observed from 1690 to $1260 \mathrm{cp}$, following US exposure. For PAA, a change was observed from 785 to $725 \mathrm{cp}$ at $\mathrm{pH} 3$ and from 1740 to $1550 \mathrm{cp}$ at $\mathrm{pH}$ 5. When the shear viscosity was measured at $\mathrm{pH} 10$ for the PAA solution, a value of $2320 \mathrm{cp}$ indicated that no change occurred in the shear viscosity upon US exposure. The polymer condition at $\mathrm{pH} 10$ could be explained by the presence of electrostatic repulsions between the $\mathrm{COO}^{-}$groups of PAA, thus leading to a higher viscosity than the other samples at $\mathrm{pH} 3$ and 5. Therefore, the PAA polymer chains were considered to be more extended and to have fewer hydrogen bonds at $\mathrm{pH} 10$.

\section{Effect of US absorption on the aqueous polymer medium}

Because these changes in the shear viscosity due to US were observed in aqueous solutions of polymers with different chemical structures, the US intensity was monitored both before and after transmittance of the US through the sample solution to analyze US absorption by the aqueous polymer solutions. Here the transmittance of US through the aqueous solution was investigated using a US detector to measure US intensity. Thus, the US intensity was attenuated from $I_{0}$ to $I_{\mathrm{US}}$ when the US passed through the sample medium in a glass cell having $L=30 \mathrm{~mm}$, containing $60 \mathrm{ml}$ total volume of the aqueous polymer solution at different polymer concentrations. The US intensity decreased from $I_{0}$ to $I_{\mathrm{US}}$ when the experiments were performed without and then with polymer in solution. The peak heights of the US profiles were measured by oscilloscope after the US intensity was received by the probe. Then, $I_{0}$ and $I_{\mathrm{US}}$ were observed both before and after US transduction at each aqueous polymer solution concentration. The transmittance $t=I_{\mathrm{US}} / I_{0}$ (1) was calculated for different polymer solutions. Results show that the polymer solution exhibited a 

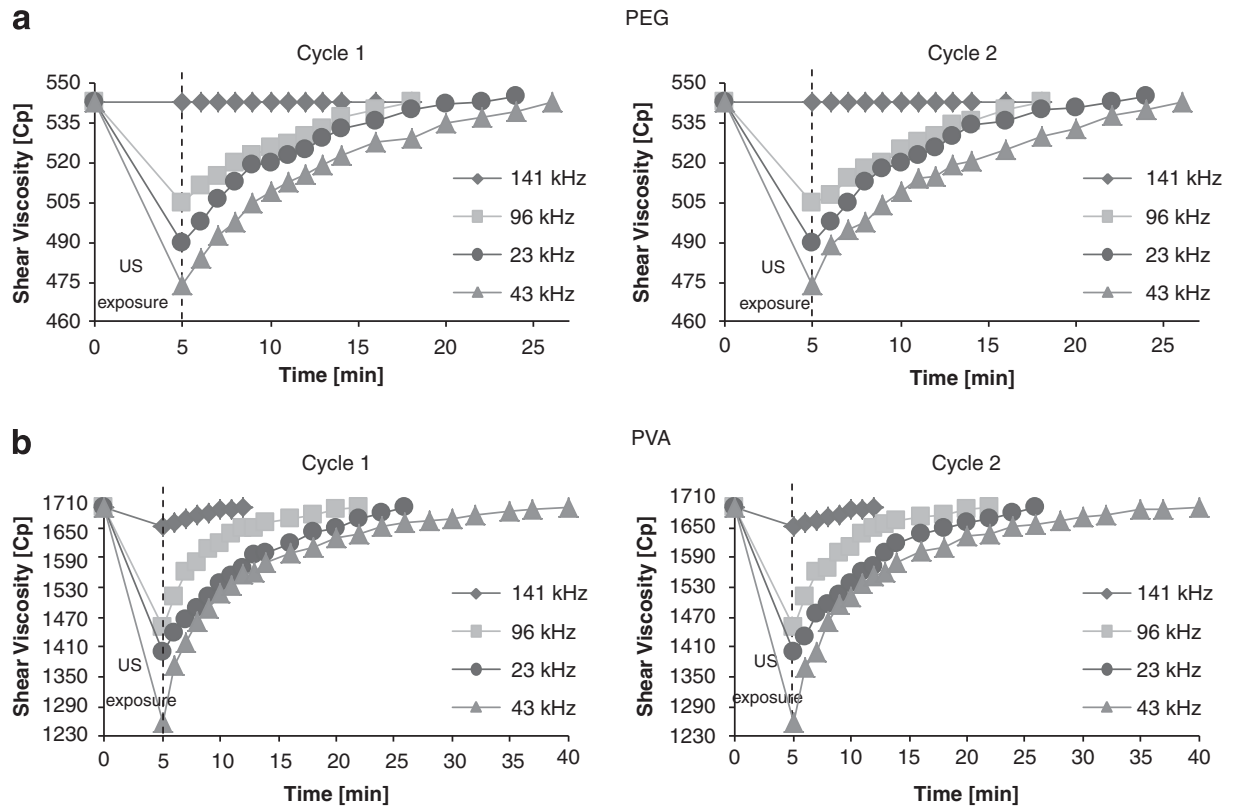

C

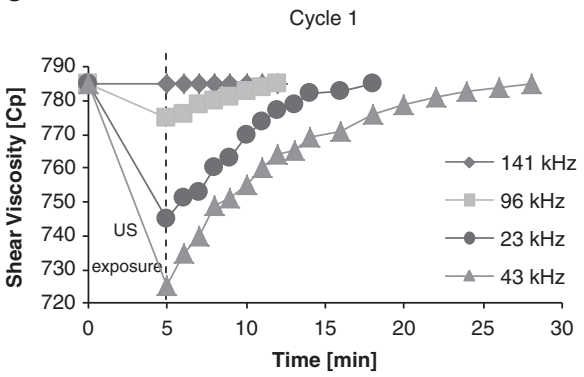

d

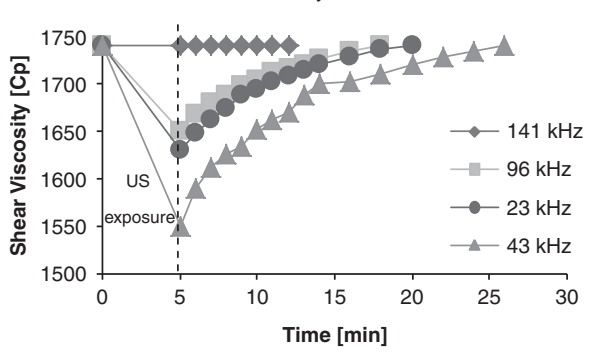

e

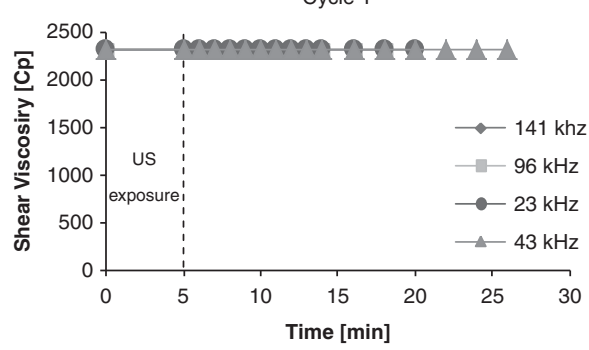

PAA at $\mathrm{pH} 3$

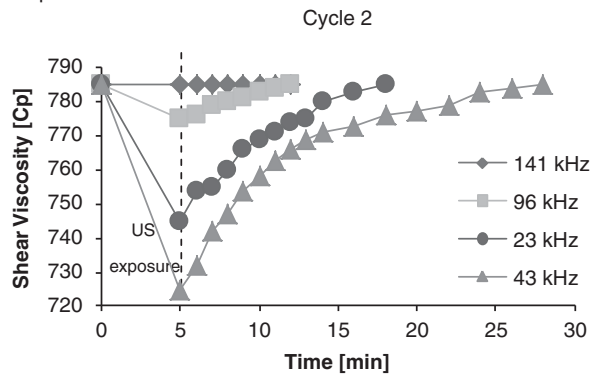

PAA at $\mathrm{pH} 5$

Cycle 2

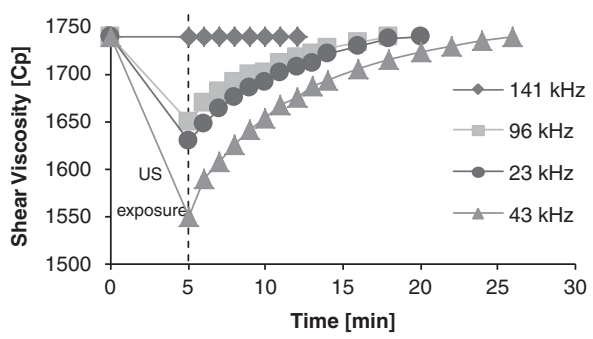

PAA at $\mathrm{pH} 10$

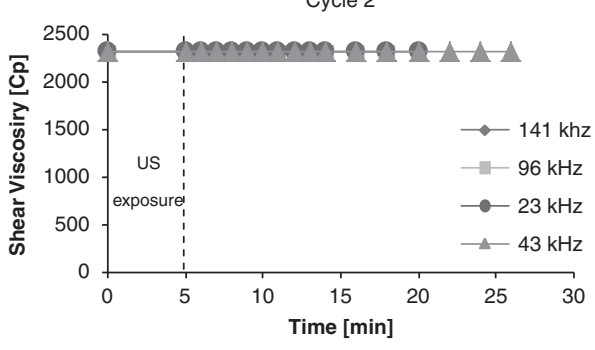

Figure 1 Change in the shear viscosity for aqueous (a) PEG, (b) PVA, (c) PAA at pH 3, (d) PAA at pH 5 and (e) PAA at pH 10 with a 10 wt\% concentration. The US power dissipated was 8.5-9 W. 


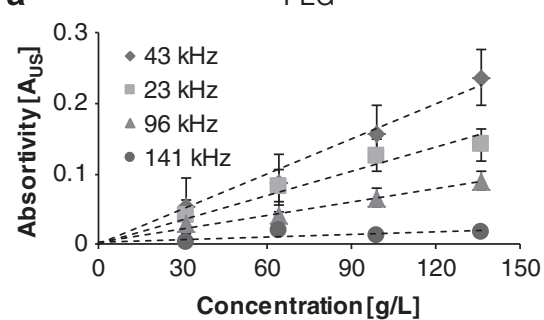

C

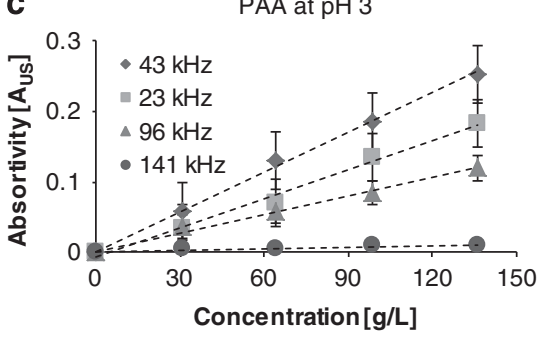

b

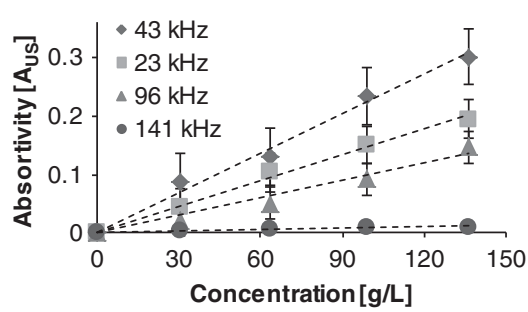

d

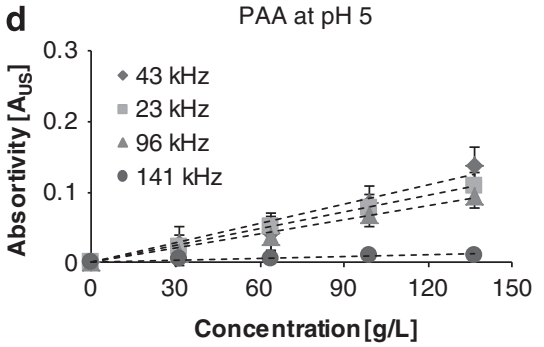

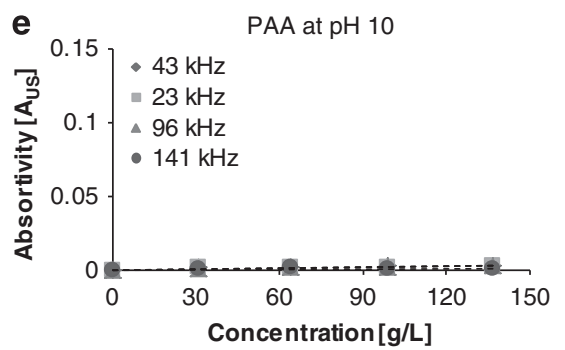

Figure 2 US absorptivity ( $A_{U S}=\varepsilon L C$ ) of for (a) PEG, (b) PVA, (c) PAA at pH 3, (d) PAA at pH 5, and (e) PAA at pH 10 with different concentrations. The US power dissipated was adjusted to $8.5-9 \mathrm{~W}$.

Table 1 Eus coefficient $\left(\mathrm{m}^{2} \mathrm{~g}^{-1}\right)$ for aqueous polymer solutions at different frequencies

\begin{tabular}{lccccc}
\hline $\begin{array}{l}\text { Frequency } \\
(\mathrm{kHz})\end{array}$ & $\begin{array}{c}\text { PEG at } \\
p H 3\end{array}$ & $\begin{array}{c}\text { PVA at } \\
\text { 3H 3 }\end{array}$ & $\begin{array}{c}\text { PAA at } \\
p H 3\end{array}$ & $\begin{array}{c}\text { PAA at } \\
p H 5\end{array}$ & $\begin{array}{c}\text { PAA at } \\
p H 10\end{array}$ \\
\hline 43 & $5.3 \times 10^{-5}$ & $7.6 \times 10^{-5}$ & $6.3 \times 10^{-5}$ & $3.0 \times 10^{-5}$ & $3.0 \times 10^{-6}$ \\
23 & $3.3 \times 10^{-5}$ & $5.0 \times 10^{-5}$ & $4.3 \times 10^{-5}$ & $2.6 \times 10^{-5}$ & $3.0 \times 10^{-6}$ \\
96 & $2.3 \times 10^{-5}$ & $3.3 \times 10^{-5}$ & $3.0 \times 10^{-5}$ & $2.3 \times 10^{-5}$ & $3.0 \times 10^{-6}$ \\
141 & $3.3 \times 10^{-6}$ & $3.0 \times 10^{-6}$ & $3.0 \times 10^{-6}$ & $3.0 \times 10^{-6}$ & $3.3 \times 10^{-7}$ \\
\hline
\end{tabular}

Abbreviations: PAA, polyacrylic acid; PEG, polyethylene glycol; PVA, polyvinyl alcohol; US, ultrasound.

tendency to show that $I_{0}>I_{\mathrm{US}}$, meaning that the US transmittance decreased at each frequency as a function of US absorption. To examine the degree of US absorbed by the aqueous polymer solution, we defined US absorption as $A_{\mathrm{US}}$

$$
A_{\mathrm{US}}=-\log _{10}(t)=\log _{10}\left(I_{0} / I_{\mathrm{US}}\right)
$$

The absorptivity of the US in the solution is expected to increase continuously with increasing polymer concentration. Notably, the experimental data showed a linear correlation in each polymer system. Therefore, the absorptivity of the US can be expressed as $A_{\mathrm{US}}=\varepsilon_{\mathrm{US}} L C$, where $L$ is the glass cell length $(30 \mathrm{~mm})$ and $\varepsilon_{\mathrm{US}}$ is the constant slope. The value $\varepsilon_{U S}$ represents the area of US absorptivity in the solution medium per unit weight at the transduced distance $\left(\mathrm{Lg}^{-1} \mathrm{~cm}^{-1}\right)$. Therefore, we were able to perform the following conversion of the absorptivity, $\varepsilon_{\text {US }}$, given in units of centimeters and grams per liter: $\mathrm{Lg}^{-1} \mathrm{~cm}^{-1}=0.1 \mathrm{~m}^{2} \mathrm{~g}^{-1}$. This unit constant indicates that the value of $\varepsilon_{U S}$ is the US absorption area for the unit weight of each polymer solution.

In Figure 2, the slope in the equation increased at a US of $43 \mathrm{kHz}$ for PEG, PVA and PAA at $\mathrm{pH}$ 3. The resultant $\varepsilon_{\mathrm{US}}$ values are listed in Table 1 . The trend increased in order of $43>23>96>141 \mathrm{kHz}$ for PEG, PVA and PAA at $\mathrm{pH} 3$. For example, at $43 \mathrm{kHz}$, the values of $\varepsilon_{\mathrm{US}}$ were $7.6 \times 10^{-5}, 6.3 \times 10^{-5}$ and $5.3 \times 10^{-5} \mathrm{~m}^{2} \mathrm{~g}^{-1}$ for PVA, PAA (at $\mathrm{pH} 3$ ) and PEG, respectively. The value for PVA represented a higher absorptivity relative to the others, indicating that PVA solutions could adsorb more US. However, the PAA samples at $\mathrm{pH} 5$ and 10 possessed different US absorptivities, even though the same polymer solution was used at different pHs.

Relative to the viscosity at $\mathrm{pH} 3$, a high value for viscosity was observed at $\mathrm{pH}$ 10. This outcome was due to the extension of PAA chains because of the electrostatic effects of charged PAA. However, Figure 2 showed that the results for $\varepsilon_{U S}$ were very low at $\mathrm{pH} 10$ for the PAA solution. This result seemed to suggest very low absorption of US in such a viscous solution as that of charged PAA. Therefore, very little US absorption occurred in the dissociated PAA without $\mathrm{OH}$ groups, although high viscosity was observed in the aqueous PAA solution at $\mathrm{pH} 10$.

\section{Effect of US on the change in the FT-IR spectra of aqueous polymers}

To clarify the change in the shear viscosity and the level of US absorption in each aqueous polymer solution both before and after US exposure, FT-IR spectra were obtained for the aqueous polymer 
a

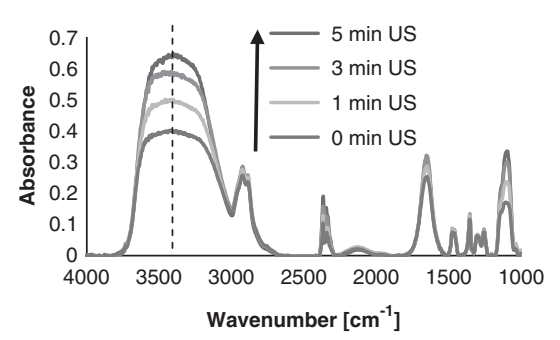

c

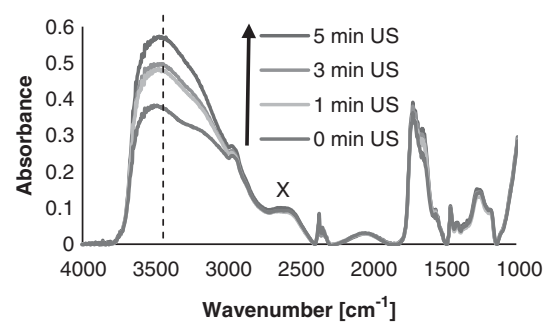

b

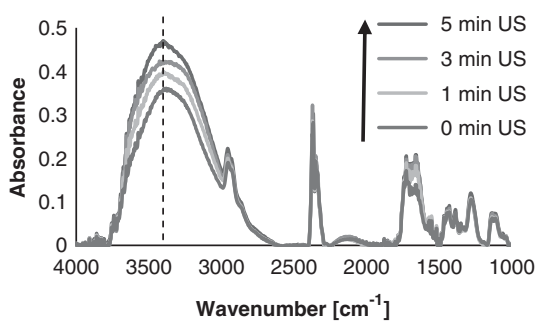

d

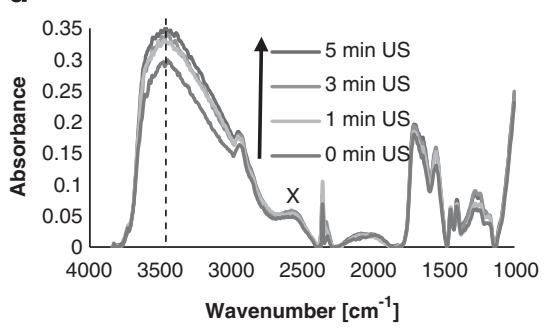

e

PAA at $\mathrm{pH} 10$

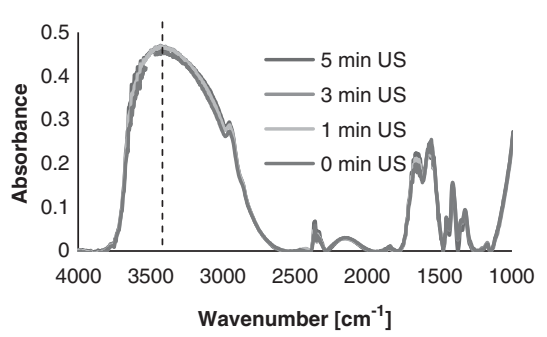

Figure 3 FT-IR spectra before and after US exposure at different times for (a) PEG, (b) PVA, (c) PAA at pH 3, (d) PAA at pH 5 and (e) PAA at pH 10 at a 10 wt $\%$ concentration.

solutions. The experimental method was similar to previous reports for water-soluble polysaccharides ${ }^{13}$ and water-swelled hydrophilic microgels. ${ }^{10}$ In the present experiments, for PEG, PVA and PAA, a small amount of polymer solution was dropped onto the $\mathrm{CaF}_{2}$ windows, and the sandwiched windows were then placed into the water bath of the sonoreactor to be exposed to US. FT-IR measurements were performed immediately after US exposure was terminated. It was confirmed that chemical adsorption of polymer to the $\mathrm{CaF}_{2}$ windows did not occur after the FT-IR measurements were finished. This phenomenon was checked by washing the windows after US exposure.

Figure 3 shows the FT-IR spectra of the aqueous polymers for (a) PEG, (b) PVA, (c) PAA at pH 3, (d) PAA at pH 5 and (e) PAA at pH 10 both before and after various US exposures were performed for 1 , 3 and $5 \mathrm{~min}$. The FT-IR spectra exhibited the characteristic bands for PEG and PVA at $3000-3800 \mathrm{~cm}^{-1} .{ }^{27-29}$ FT-IR peaks for $\mathrm{PEG}^{27,28}$ and $\mathrm{PVA}^{29}$ in water were assigned at $3300-3500 \mathrm{~cm}^{-1}$ for $\mathrm{OH}$ stretching of the polymers and water molecules. For PAA, the $\mathrm{OH}$ stretching band was noted at $2500-3800 \mathrm{~cm}^{-1} \cdot 30$ For the monomeric acid $\mathrm{OH}$ at $3500-3800 \mathrm{~cm}^{-1}$ and the hydrogen-bonded acid $\mathrm{OH}$ at $2500-3500 \mathrm{~cm}^{-1}$, a characteristic band for each polymer was observed in these regions. ${ }^{31} \mathrm{~A}$ non-hydrogen-bonded peak for the polymer chain and water molecules appeared in the $3400-3800 \mathrm{~cm}^{-1}$ region, and strong interactions between the polymer chain and water were observed in the $3000-3400 \mathrm{~cm}^{-1}$ region due to hydrogen bonding. In the $2500-2600 \mathrm{~cm}^{-1}$ region, Max et al. ${ }^{32}$ reported that the carboxylic acid groups are hydrogen bonded to water, which is indicated by ' $\mathrm{X}$ ' in the spectra in Figures $3 c$ and d. Furthermore, carboxylic dimers display an $\mathrm{OH}$ stretching band in the region of $2500-3300 \mathrm{~cm}^{-1}$, which is generally centered near $3000 \mathrm{~cm}^{-1} .^{33}$ Similar results for inter- and intra-hydrogen-bonded bands for PAA were reported. ${ }^{31-34}$ In addition, a band observed at $2800-3000 \mathrm{~cm}^{-1}$ was attributed to $\mathrm{CH}$ stretching from alkyl groups. ${ }^{28,31}$ The wavenumber region between 1500 and $1800 \mathrm{~cm}^{-1}$ corresponded to a $\mathrm{C}=\mathrm{O}$ group, and the peak at $1640 \mathrm{~cm}^{-1}$ corresponded to $\mathrm{OH}$ bending. In PAA at $\mathrm{pH} 3$, the carbonyl band was centered at $1710 \mathrm{~cm}^{-1}$. When PAA was tested at $\mathrm{pH} 5$, there were two peaks centered at 1710 and $1540 \mathrm{~cm}^{-1}$ for the carbonyl bands..$^{35,36}$ This result indicated that the carboxylic acid group was partially dissociated: $-\mathrm{COOH} \rightleftarrows-\mathrm{COO}^{-}+\mathrm{H}^{+}$. For PAA at $\mathrm{pH} \mathrm{10,} \mathrm{it} \mathrm{was} \mathrm{interesting} \mathrm{to} \mathrm{note} \mathrm{that} \mathrm{the} \mathrm{OH}$ band shifted toward a lower wavenumber region of $3200-3400 \mathrm{~cm}^{-1}$ (Figure 4). Nevertheless, the spectral change caused by US exposure was less than that of the PAA solution at $\mathrm{pH} 3$. For PAA solutions at $\mathrm{pH} 3$ and 5, dimer formation of $-\mathrm{COOH}$ groups was observed in the $2400-2700 \mathrm{~cm}^{-1}$ region (Figure 5). However, for $\mathrm{PAA}$ at $\mathrm{pH} 10$, no dimer formation was indicated, thereby indicating dissociation of $-\mathrm{COOH} \rightarrow-\mathrm{COO}^{-}$ $+\mathrm{H}^{+}$. In addition in Figure 5, the $\mathrm{OH}$ stretching band changed for (a) PEG, (b) PVA and (c) PAA at pH 3. For further analysis, the FT-IR spectra of these aqueous polymers were deconvoluted in the $2400-3800 \mathrm{~cm}^{-1}$ region to compare their peak positions.

For PEG and PVA, $\mathrm{OH}$ and $\mathrm{CH}$ bands were found in the resulting FT-IR spectra. Therefore, these spectra were deconvoluted to consider 
a

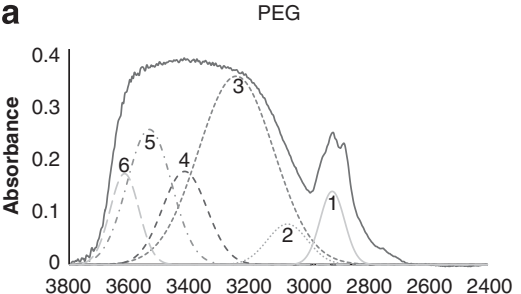

Wavenumber $\left[\mathrm{cm}^{-1}\right]$

C

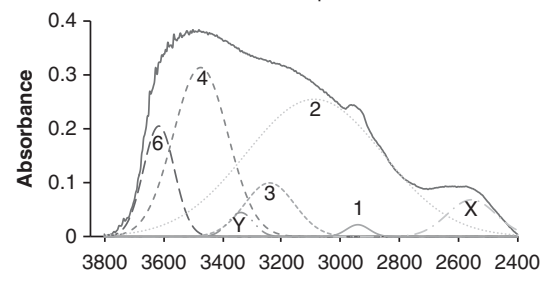

Wavenumber $\left[\mathrm{cm}^{-1}\right]$ b PVA

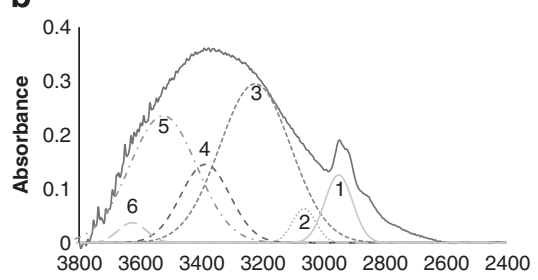

Wavenumber $\left[\mathrm{cm}^{-1}\right]$

d

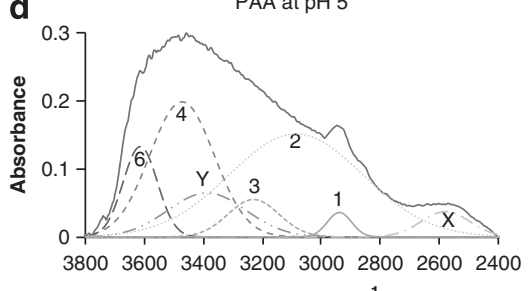

Wavenumber $\left[\mathrm{cm}^{-1}\right]$

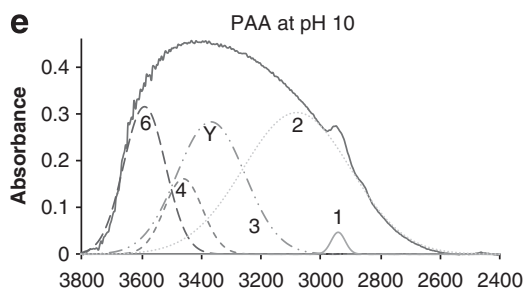

Wavenumber $\left[\mathrm{cm}^{-1}\right]$

Figure 4 Comparison of the deconvoluted $\mathrm{OH}$ stretching band of (a) PEG, (b) PVA and (c) PAA at pH 3, (d) PAA at pH 5 and (e) PAA at pH 10 without US exposure. Peak 1 was assigned to $\mathrm{CH}$ stretching at $2927-2939 \mathrm{~cm}^{-1}$.
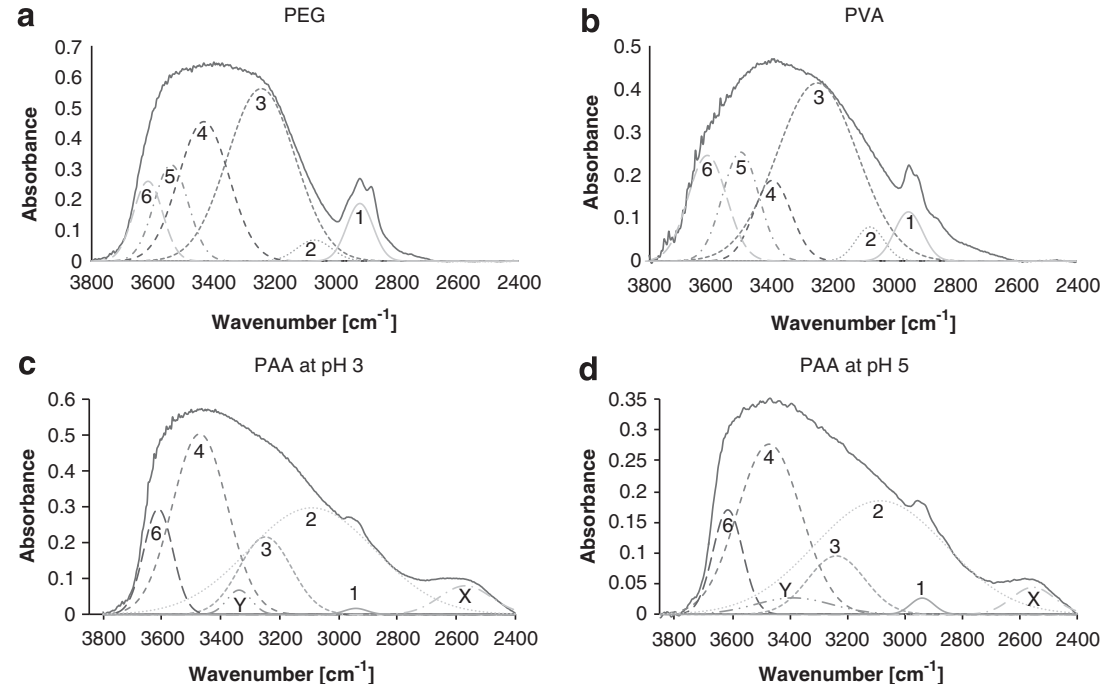

Wavenumber $\left[\mathrm{cm}^{-1}\right]$

Wavenumber $\left[\mathrm{cm}^{-1}\right]$

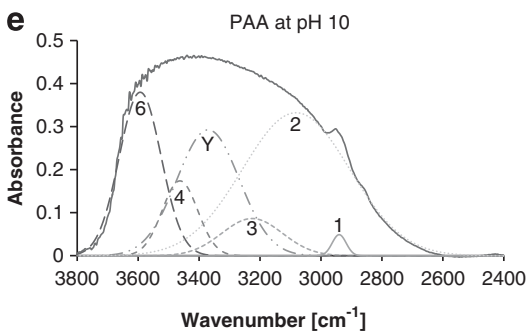

Figure 5 Comparison of the deconvoluted $\mathrm{OH}$ stretching band of (a) PEG, (b) PVA, (c) PAA at pH 3, (d) PAA at pH 5 and (e) PAA at pH 10 after 5 min of US exposure. Peak 1 was assigned to $\mathrm{CH}$ stretching at $2927-2939 \mathrm{~cm}^{-1}$. 
both stretching bands in the wavenumber region. The spectra were decomposed into Gaussian components by curve fitting. ${ }^{13,27}$ The peak center results are summarized in Table 2. Figure 4 displays the fixed curve of the $\mathrm{OH}$ stretching of aqueous polymer without US exposure for (a) PEG, (b) PVA and (c) PAA at $\mathrm{pH}$ 3. In addition, the fixed curve of $\mathrm{CH}$ stretching was included in the spectra. For the deconvoluted spectra, peak 1 was assigned to the $\mathrm{CH}$ stretching bands. ${ }^{28,31}$ Peak 2 was assigned to the strong hydrogen-bond interactions between water and the $\mathrm{OH}$ groups from the polymer chain. ${ }^{27}$ Here, for PEG and PVA, peaks 3 and 4 were assigned to the strong hydrogen-bond interactions between the polymer and

Table 2 Centered peak wavenumber determined by curve fitting for the $\mathrm{OH}$ stretching band

\begin{tabular}{|c|c|c|c|c|c|c|c|c|c|c|}
\hline \multirow{2}{*}{$\begin{array}{l}\text { US } \\
\text { peak }\end{array}$} & \multicolumn{2}{|c|}{$\begin{array}{c}\text { PEG at } \\
p H 3\end{array}$} & \multicolumn{2}{|c|}{$\begin{array}{c}\text { PVA at } \\
p H 3\end{array}$} & \multicolumn{2}{|c|}{$\begin{array}{c}\text { PAA at } \\
p H 3\end{array}$} & \multicolumn{2}{|c|}{$\begin{array}{c}P A A \text { at } \\
\text { pH } 5\end{array}$} & \multicolumn{2}{|c|}{$\begin{array}{l}\text { PAA at } \\
\text { pH } 10\end{array}$} \\
\hline & $O \min$ & $5 \mathrm{~min}$ & $O \min$ & $5 \mathrm{~min}$ & $O \min$ & $5 \mathrm{~min}$ & $O \min$ & $5 \mathrm{~min}$ & $O \min$ & $5 \mathrm{~min}$ \\
\hline$x$ & & & & & 2548 & 2555 & 2546 & 2546 & & \\
\hline 1 & 2921 & 2919 & 2948 & 2949 & 2927 & 2927 & 2939 & 2939 & 2941 & 2939 \\
\hline 2 & 3071 & 3068 & 3061 & 3075 & 3092 & 3092 & 3048 & 3054 & 3080 & 3080 \\
\hline 3 & 3243 & 3242 & 3221 & 3248 & 3249 & 3249 & 3228 & 3239 & 3226 & 3223 \\
\hline Y & & & & & 3338 & 3338 & 3390 & 3387 & 3365 & 3368 \\
\hline 4 & 3415 & 3430 & 3384 & 3395 & 3473 & 3453 & 3497 & 3473 & 3484 & 3461 \\
\hline 5 & 3534 & 3537 & 3522 & 3499 & & & & & & \\
\hline 6 & 3617 & 3613 & 3623 & 3608 & 3615 & 3612 & 3633 & 3639 & 3630 & 3591 \\
\hline
\end{tabular}

Abbreviations: PAA, polyacrylic acid; PEG, polyethylene glycol; PVA, polyvinyl alcohol; US, ultrasound.
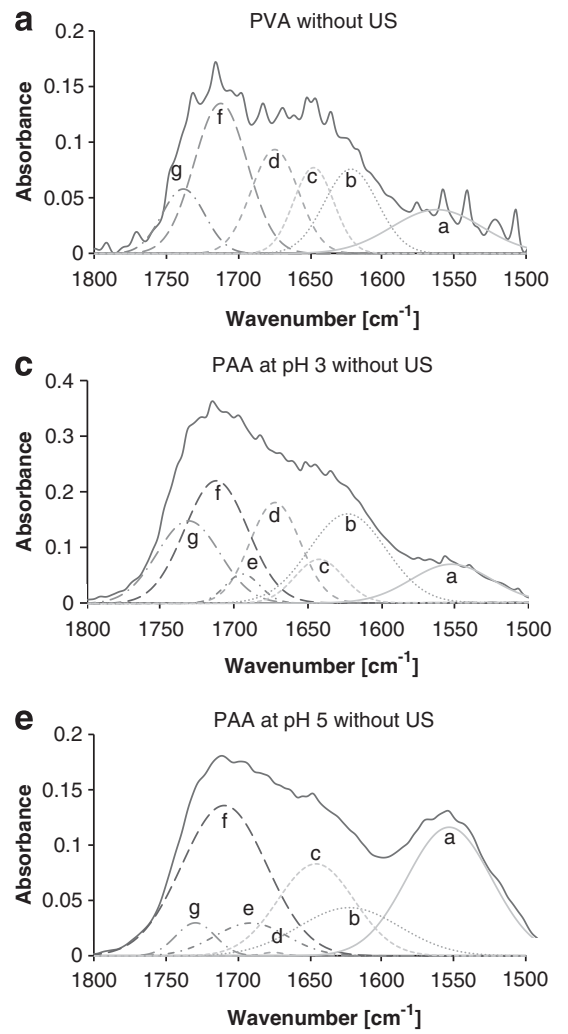

water molecules and to the weak hydrogen-bond interactions between the polymer and water molecules, respectively. ${ }^{28,29}$ Peaks 5 and 6 were assigned to free $\mathrm{OH}$ groups from either the polymer or water molecules and to those from free water molecules, respectively. ${ }^{29,30,37}$

For PAA, peaks 2 and $\mathrm{X}$ corresponded to the PAA dimer interactions, and an additional overtone of approximately 2650 and $2550 \mathrm{~cm}^{-1}$ (Silver stein et al. ${ }^{35}$ ) indicated the presence of strong hydrogen-bond interactions between $-\mathrm{COOH}$ groups. Peak $\mathrm{Y}$ was assigned to weak hydrogen-bond interactions. ${ }^{32,36,38}$ Figure 5 also contains the fixed curves of (a) PEG, (b) PVA, (c) PAA at pH 3, (d) $\mathrm{PAA}$ at $\mathrm{pH} 5$ and (e) PAA at $\mathrm{pH} 10$ for data regarding US exposure.

Comparison of (a) PEG with Figures $4 \mathrm{a}$ and $5 \mathrm{a}$ showed that peak 1 for the $\mathrm{CH}$ band and peak 2 for the hydrogen bonding of water-OH group underwent less change. However, the intensity of peak 5 decreased, whereas peak 4 became intense, after US exposure. The change in Figure 5a) meant that free $\mathrm{OH}$ groups of PEG could bind with water at the end of the polymer chain. For PVA having side $\mathrm{OH}$ groups, it was similarly observed that the intensity of peak 5 decreased, whereas peak 6 increased as a result of US exposure. This result could explain the US-enhanced breakage of the interactions between water molecules and the $\mathrm{OH}$ groups of PVA.

In the case of (c) PAA at $\mathrm{pH} 3$, the band intensities of peaks 4 and 6 were intense following US exposure. However, hydrogen bonds due to the $-\mathrm{COOH}$ group in the dimer at approximately $3000-3200 \mathrm{~cm}^{-1}$ were significantly changed. This result indicated that US was effective in breaking the $-\mathrm{COOH}$ dimers and increased the population of free $\mathrm{OH}$ groups from $-\mathrm{COOH}$ or water molecules. At $\mathrm{pH} 5$ and 10 , the population of each peak changed less before and after US exposure. The insignificant change at $\mathrm{pH} 10$ could be due to the full dissociation
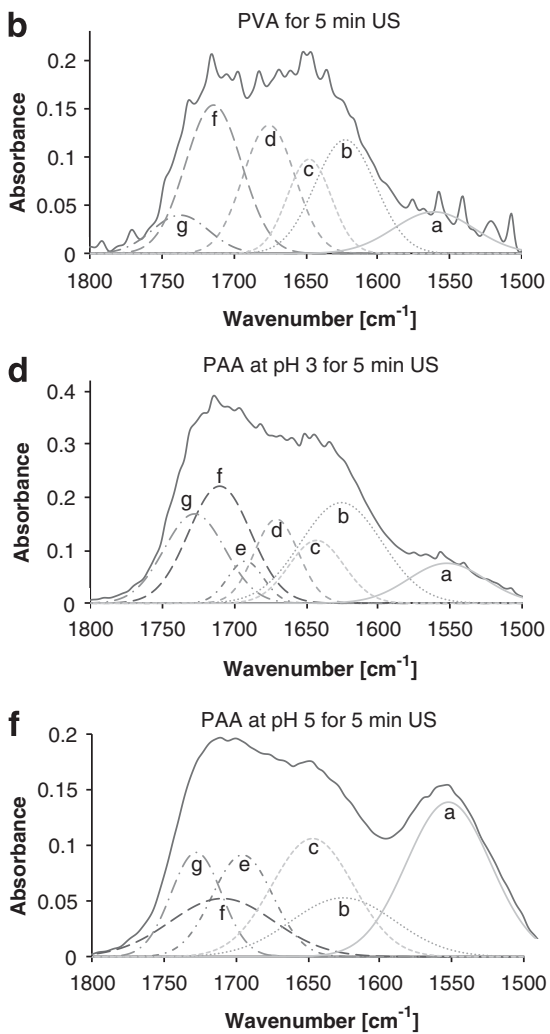

Figure 6 Comparison of the deconvoluted $\mathrm{C}=\mathrm{O}$ stretching bands of PVA, PAA at $\mathrm{pH} 3$ and PAA at $\mathrm{pH} 5$ before (a, c, e) and after 5 min of US treatment (b, d, f). 
a

PEG

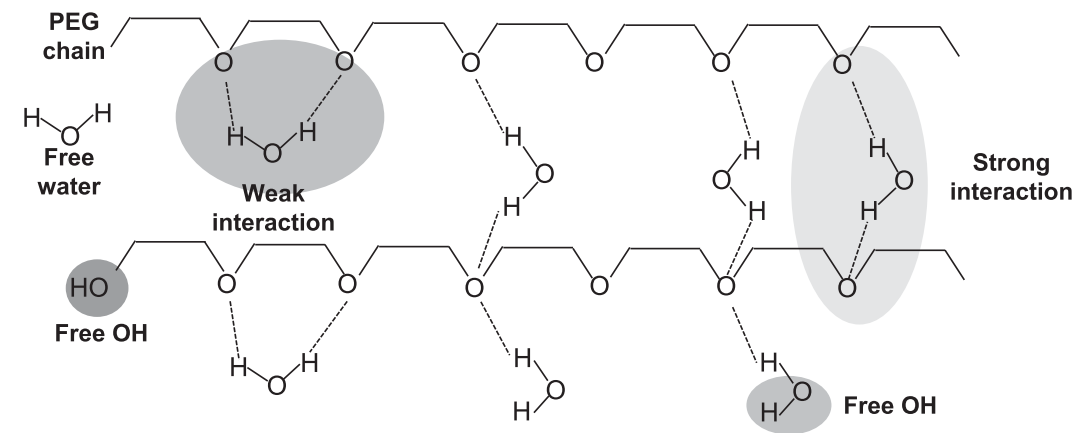

b

PVA

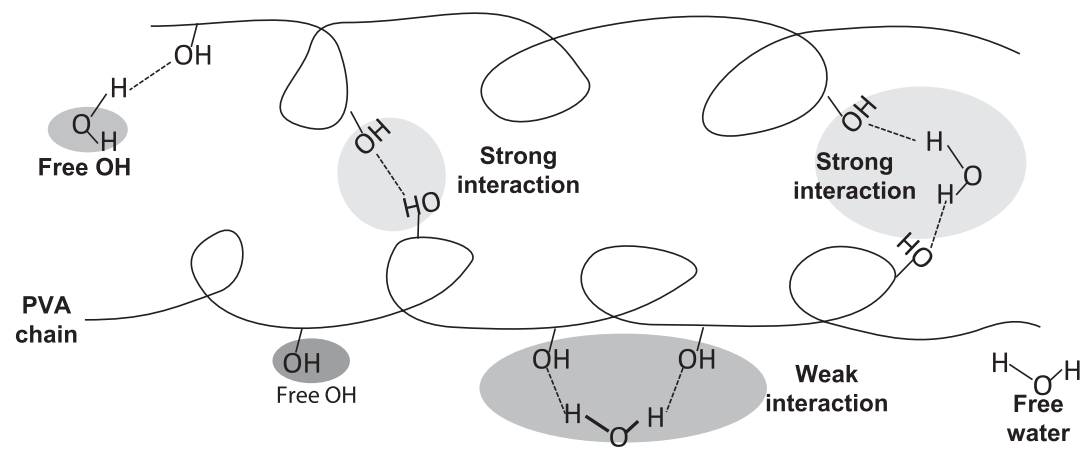

C

PAA

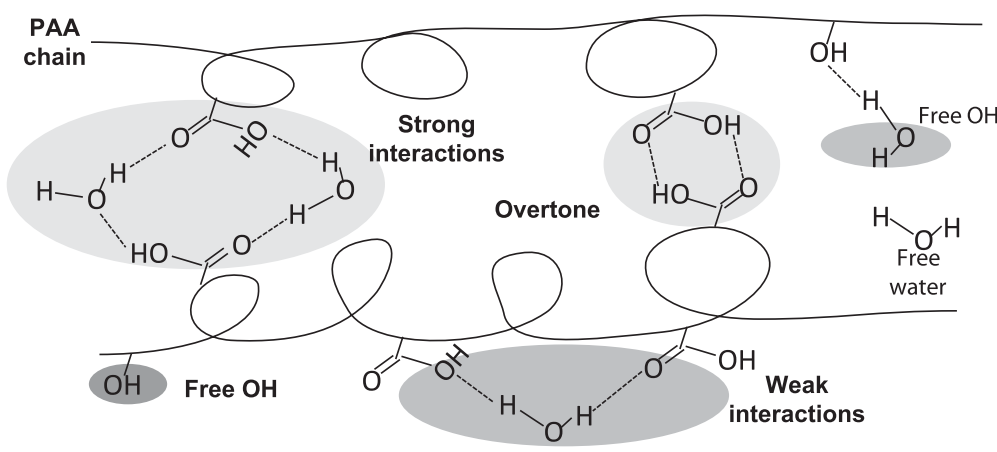

Scheme 2 Hydrogen-bond cross-linked structures of (a) PEG, (b) PVA and (c) PAA with water. A full color version of this figure is available at Polymer Journal online.

Table 3 Centered peak wavenumber determined by curve fitting for the $\mathbf{C}=\mathbf{O}$ stretching band

\begin{tabular}{|c|c|c|c|c|c|c|}
\hline \multirow[b]{2}{*}{ US peak } & \multicolumn{2}{|c|}{$P V A$} & \multicolumn{2}{|c|}{ PAA at $p H 3$} & \multicolumn{2}{|c|}{$P A A$ at $p H 5$} \\
\hline & Omin & $5 \mathrm{~min}$ & $O \min$ & $5 \mathrm{~min}$ & Omin & $5 \mathrm{~min}$ \\
\hline$a$ & 1562 & 1561 & 1552 & 1552 & 1553 & 1552 \\
\hline b & 1622 & 1623 & 1623 & 1626 & 1623 & 1626 \\
\hline c & 1648 & 1648 & 1642 & 1643 & 1646 & 1647 \\
\hline d & 1675 & 1676 & 1672 & 1671 & 1676 & \\
\hline e & & & 1694 & 1692 & 1691 & 1696 \\
\hline$f$ & 1712 & 1714 & 1712 & 1710 & 1710 & 1710 \\
\hline g & 1739 & 1738 & 1731 & 1728 & 1730 & 1728 \\
\hline
\end{tabular}

Abbreviations: PAA, polyacrylic acid; PEG, polyethylene glycol; PVA, polyvinyl alcohol; US, ultrasound. of carboxylic acid groups: $\mathrm{COOH} \rightarrow \mathrm{COO}^{-}+\mathrm{H}^{+}$. Furthermore, the $1700 \mathrm{~cm}^{-1}$ band for $\mathrm{C}=\mathrm{O}$ stretching contains useful information about the hydrogen-bonded and non-hydrogen-bonded carboxyl groups of PAA. ${ }^{36}$ Therefore, analysis similar to that of the $\mathrm{C}=\mathrm{O}$ band was performed with the $\mathrm{OH}$ band in Figures 4 and 5. Figure 6 shows the FT-IR spectra observed near the $1700 \mathrm{~cm}^{-1}$ region. In the $\mathrm{C}=\mathrm{O}$ stretching region, the curve fitting spectra of the linear oligomer, cyclic dimer, side-on dimer and monomeric side-chain carboxylic groups were obtained for PAA at 1676, 1692, 1710 and $1730 \mathrm{~cm}^{-1}$, respectively. The peaks at 1648 and $1622 \mathrm{~cm}^{-1}$ were assigned to water molecules interacting with PAA, ${ }^{39,40}$ and the peak at $1562 \mathrm{~cm}^{-1}$ corresponded to dissociation of carboxylic acid groups. ${ }^{38}$ Table 3 also lists each band in the FT-IR spectra both without and with US exposure in the range of $1500-1800 \mathrm{~cm}^{-1}$. By increasing the 


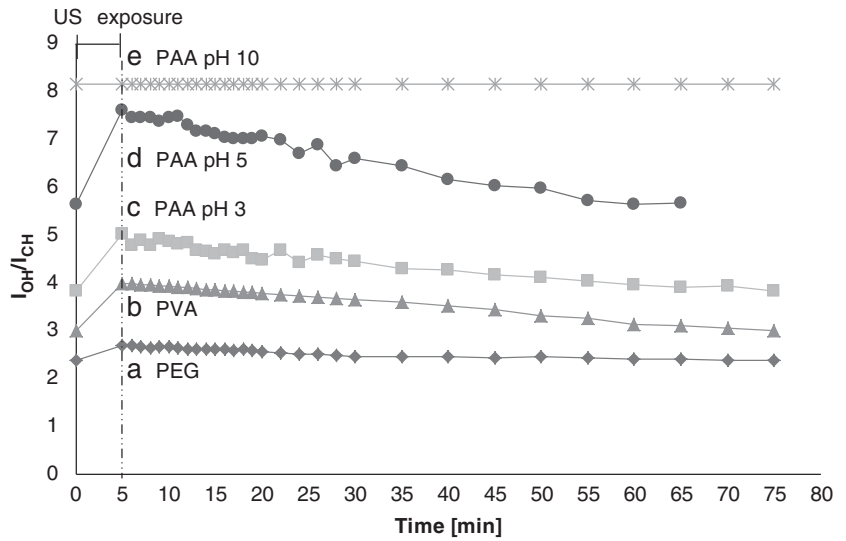

Figure 7 Change in the ratio of $\mathrm{IOH}_{\mathrm{H}} / \mathrm{I}_{\mathrm{CH}}$ intensity as a function of time observed in the FT-IR spectra of (a) PEG ( ), (b) PVA ( $\mathbf{\Delta}$ ), (c) PAA at pH 3 (西), (d) PAA at pH $5(\bullet)$ and (e) PAA at pH $10\left(^{*}\right)$.

US exposure time from 1 to $5 \mathrm{~min}$, the intensity of the $1690 \mathrm{~cm}^{-1}$ band increased. This result indicated that water molecules could associate with the $\mathrm{C}=\mathrm{O}$ group in PAA at $\mathrm{pH} 3$ following US exposure. A similar tendency was observed in the spectrum at $\mathrm{pH} 5$ for partial dissociation of $\mathrm{COO}^{-}$in PAA, as shown in Figure 3b. A similar result was also observed in PVA at $1675 \mathrm{~cm}^{-1}$ for the -O$\mathrm{C}=\mathrm{O}$ group, which remained intact without hydrolysis of the acyl acetate group. Altogether, these data indicated that US exposure enhanced water solvation to the $-\mathrm{C}=\mathrm{O}$ group in the polymer (Scheme 2). Figure 7 shows the change in the ratio of the $I_{\mathrm{OH}} / I_{\mathrm{CH}}$ intensity as a function of time in the FT-IR spectra for (a) PEG, (b) PVA, (c) PAA at $\mathrm{pH} 3$, (d) PAA at $\mathrm{pH} 5$ and (e) PAA at $\mathrm{pH}$ 10. After US exposure ceased, the values of the peak ratios in the FT-IR spectra obtained at $\mathrm{pH} 3$ and 5 increased. This outcome resulted from the attribution of the $\mathrm{OH}$ group to the free $\mathrm{COOH}$ group or water molecule, during US exposure. Notably, the values of $I_{\mathrm{OH}} / I_{\mathrm{CH}}$ decreased gradually and returned to their original values within $60 \mathrm{~min}$, when US exposure ceased. However, no change occurred at $\mathrm{pH} 10$, even though US exposure occurred. Consequently, the hydrogen bonds were reformed, and the aqueous polymer solution became viscous again, as shown in Figure 1. It was reasonable to conclude that US exposure disrupted the hydrogen bonds of the aqueous polymer solutions of PEG, PVA and PAA at pH 3 and 5 due to US absorption and could enhance water solvation into the polymer.

As mentioned, the present paper could provide the first results of the effect of US on different chemical structures of water-soluble polymers, which exhibited similar amounts of US absorption to form free $\mathrm{OH}$ groups in water following US exposure, as shown in Scheme 2.

\section{CONCLUSION}

The present paper concluded that US, especially at $43 \mathrm{kHz}$, induced a change in the shear viscosity due to the disruption of hydrogen bonds by efficient US absorption by the aqueous medium, and due to the formation of free water with $\mathrm{OH}$ groups and of $\mathrm{OH}$ groups in the water-soluble polymers. We also evaluated the values of US absorptivity for $\varepsilon_{\text {US }}$, which were $5.3 \times 10^{-5}, 7.6 \times 10^{-5}, 6.3 \times 10^{-5}$, $3.0 \times 10^{-5}$ and $3.0 \times 10^{-6}$ for PEG, PVA, PAA at $\mathrm{pH} 3, \mathrm{PAA}$ at $\mathrm{pH}$ 5 and PAA at $\mathrm{pH} 10$, respectively, at a frequency of $43 \mathrm{kHz}$. US exposure disrupted the hydrogen bonds in the polymers, especially at the $\mathrm{OH}$ groups, and also enhanced the solvation of water to each $\mathrm{OH}$ and $\mathrm{C}=\mathrm{O}$ group.

1 Mason, T. J. \& Lorimer, J. P. Applied Sonochemistry: The Uses of Power Ultrasound in Chemistry and Processing (Wiley-VCH Verlag GmbH, Weinheim, 2002).

2 Price, G. J. Current Trends in Sonochemistry (Royal Society of Sonochemistry, Thomas Graham House, Science Park, Cambridge, 1992).

3 Eldik, R. \& Hubbard, C. D. Chemistry Under Extreme or Non-classical Conditions (Wiley \& Sons, New York, 1996).

4 Mason, T. J., Paniwnyls, L. \& Lorimer, J. P. The uses of ultrasound in food technology. Ultrason. Sonochem. 3, s253-s260 (1996).

5 Chermat, F. \& Hoarau, N. Hazard analysis and critical control point (HACCP) for an ultrasound food processing operation. Ultrason. Sonochem. 11, 257-260 (2004).

6 Roy, D., Cambre, J. N. \& Sumerlin, B. S. Future perspectives and recent advances in stimuli-responsive materials. Prog. Polym. Sci. 36, 278-301 (2010).

7 Hyun-Jong Kim, H., Matsuda, H. \& Zhou, I. Honma, ultrasound-triggered smart drug release from a poly(dimethylsiloxane)-mesoporous silica composite. Adv. Mater. 18, 3083-3088 (2006)

8 De Geest, B. G., Skirtach, A. G., Mamedov, A. A., Antipov, A. A., Kotov, N. A., De Smedt, S. C. \& Sukhorukov, G. B. Ultrasound-triggered release from multilayered capsules. Small 3, 804-808 (2007).

9 Huo, D. \& Kobayashi, T. Sonoresponsive volume phase-transition behavior of crosslinked poly(n-isopropylacrylamide-co-acrylic acid). Microgel. Chem. Lett. 35, 776-777 (2006).

10 Venegas-Sanchez, J. A., Kusunoki, T., Yamamoto, M. \& Kobayashi, T. Sonoresponsive volume phase-transition behavior of crosslinked poly(n-isopropylacrylamide-coacrylic acid). Ultrason. Sonochem. 20, 1271-1275 (2013).

$11 \mathrm{Ngoc}$, N. L. \& Kobayashi, T. Ultrasound stimuli on viscometric change of aqueous copolymers having acrylic acid and $\mathrm{N}$-isopropyl acrylamide for thermo-sensitive segments. Ultrason. Sonochem. 18, 1185-1192 (2011).

12 Ngoc, N. L. \& Kobayashi, T. Ultrasound stimulus effect on hydrogen bonding in networked alumina and polyacrylic acid slurry. Ultrason. Sonochem. 17, 186-192 (2010).

13 Venegas-Sanchez, J. A., Tagaya, M. \& Kobayashi, T. Ultrasound effect used as external stimulus for viscosity change of aqueous carrageenans. Ultrason. Sonochem. 20, 1081-1091 (2013).

14 Haque, M. F., Fast, S. J., Yun, S. S. \& Stumpf, F. B. Absorption of ultrasound in binary solutions of polyacrylamide with water. J. Acoust. Soc. Am. 77, 2181-2183 (1985).

15 So, J. H., Sirvent, R. E., Yun, S. S. \& Stumpf, F. B. Ultrasonic absorption and velocity measurements for poly (vinyl alcohol) and water solutions. J. Acoust. Soc. Am. 96 3807-3808 (1994).

16 So, J. H., Sirvent, R. E., Yun, S. S. \& Stumpf, F. B. Ultrasonic absorption and velocity measurements for poly (acrylic acid) and water solutions. J. Acoust. Soc. Am. 98, 659-660 (1995).

17 Bae, J. R., Kim, J. K. \& Yi, M. H. Ultrasonic absorption and velocity measurements for polyethylene glycol and water solutions. Jpn. J. Appl. Phys. 39, 2946-2947 (2000)

18 Bae, J. B. Ultrasonic velocity and absorption measurements in an aqueous solution of poly (sodium 4-styrenesulfonete). Macromol. Res. 12, 559-563 (2004).

19 Finch, C. A. Chemistry and Technology of Water-soluble Polymers (Plenum Press, New York, 1983)

20 Liu, M., Cheng, R., Wu, C. \& Qian, R. Viscometric investigation of intramolecular hydrogen bonding cohesional entanglement in extremely dilute aqueous solution of poly vinyl alcohol. J. Polym. Sci. 35, 2421-2427 (1997).

21 Gao, H. W., Yang, R. J., He, J. Y. \& Yang, J. Rheological behaviors of PVA/ $\mathrm{H}_{2} \mathrm{O}$ solutions of high-polymer concentration. J. Appl. Polym. Sci. 116, 1459-1466 (2010).

22 Song, S. \& Kim, B. C. Characteristic rheological features of PVA solutions in water containing solvents with different hydration states. Polymer (Guildf) 45, 2381-2386 (2004).

23 Elena, E. Dormidontova, role of competitive PEG-water and water-water hydrogen bonding in aqueous solution PEG behavior. Macromolecules 35, 987-1001 (2002).

24 Ebagninin, K. W. Benchabane, A. \& Bekkour, K. Rheological characterization of poly(ethylene) oxide solution of different molecular weights. J. Colloid Interface Sci. 336, 360-367 (2009).

25 Mason, T. J., Lorimer, J. P. \& Bates, D. M. Quantifying sonochemistry: casting some light on a 'black art'. Ultrasonics 30, 40-42 (1992).

26 Merouani, S., Hamdaoui, O., Saoudi, F. \& Chiha, M. Influence of experimental parameters on sonochemistry dosimetries $\mathrm{KI}$ oxidation, Fricke reaction and $\mathrm{H}_{2} \mathrm{O}_{2}$ production. J. Hazard. Mater. 178, 1007-1014 (2010).

27 Kitano, H., Ichikawa, K., Ide, M. \& Fukuda, M. Fourier transform infrared study on the state of water sorbed to poly(ethylene glycol) films. Langmuir 17, 1889-1895 (2001).

28 Shephard, J. J., Bremer, P. J. \& McQuillan, J. Structure and conformation of methylterminated Poly(ethylene oxide) and water. Principal component analysis and band fitting of infrared absorptions. J. Phys. Chem. B 113, 14229-14238 (2009). 
29 Buslov, D. K., Sushko, N. I. \& Tretinnikov, O. N. IR investigation of hydrogen bonds in weakly hydrated films of poly(vinyl alcohol). Polymer Sci. Ser. A 53, 1121-1127 (2011).

30 Daniliuc, L. \& David, C. Intermolecular interactions in blends of poly(vinyl alcohol) with poly(acrylic acid): 2. Correlation between the states of sorbed water and the interactions in homopolymers and their blend. Polymer 37, 5219-5227 (1996).

31 Wang, H. Y., Kobayashi, T. \& Fuji, N. Molecular imprint membranes prepared by the phase inverse presepitation technique. Langmuir 12, 4850-4856 (1996).

32 Max, J. J. \& Chapados, C. Infrared spectroscopy of aqueous carboxylic acids: comparison between different acids and their salts. J. Phys. Chem. A 108 3324-3337 (2004).

33 Flakus, H. T. \& Stachowska, B. Asystematic study od polarized IR spectra of the hydrogen bond in formic acid crystals. Chem. Phys. 330, 231-244 (2006).

34 Dong, J., Ozaki, Y. \& Nakashima, K. Infrared, Raman and near infrared spectroscopic evidence for coexistence of various hydrogen bond forms in poly (acrylic acid). Macromolecules 30, 1111-1117 (1997).
35 Silverstein, R. M., Webster, F. X. \& Kiemle, D. J. in Spectrometric Identification of Organic Compounds. 7th edn 94-96 (John Wiley \& Sons Inc., 2005).

36 Tajiri, T., Morita, S. \& Ozaki, Y. Hydration mechanism on a poly(methacrylic acid) film studied by in situ attenuated total reflection infrared spectroscopy. Polymer (Guildf) 50, 5765-5770 (2009).

37 Maeda, Y., Ide, M. \& Kitano, H. Vibrational spectroscopic study on the structure of water in polymers systems. J. Mol. Liq. 80, 149-163 (1999).

38 Zaman, A. A., Tsuchiya, R. \& Moudgil, B. M. Adsorption of a low-molecular-weight polyacrylic acid on silica alumina, and kaolin. J. Colloid Interface Sci. 256, 73-78 (2002).

39 Walrafen, G. E. Raman spectral studies of the effects of temperature on water structure. J. Chem. Phys. 47, 114-126 (1967)

40 Snyder, M. Q., DeSisto, W. J. \& Tripp, C. P. An infrared study of the surface chemistry of lithium titanate spinel (Li4Ti5012). Appl. Surf. Sci. 253, 9336-9341 (2007). 